\title{
Removal of Pb by Adsorption of Amidoxime Group Modified Carbon Nanotubes
}

\author{
Wen-Ling TANG ${ }^{1, \text { a }}$, Lei XIE ${ }^{1, b,{ }^{*}, \text { Hui-Juan WANG }}{ }^{1, c}$, Sai WANG ${ }^{1, d}$ \\ ${ }^{1}$ University of South China, Hengyang 421001, China \\ atangwl66@163.com, bppxielei@163.com, '625051938@qq.com, d547757234@qq.com \\ ${ }^{*}$ Corresponding author
}

Keywords: Multi-walled Carbon Nanotubes(MWNTs), Amidoxime Group, Pb, Adsorption.

\begin{abstract}
Multi-walled carbon nanotubes (MWNTs) were modified by amidoxime group, characterized by fourier transform infrared spectrometer (FTIR). The adsorption experiments of $\mathrm{Pb}(\mathrm{Pb})$ on raw MWNTs and amidoxime group modified MWNTs(AO-MWNTs) were studied under different conditions. Results showed that, the adsorption capacities of $\mathrm{Pb}$ on raw MWNTs and AOMWNTs primarily increased with the increasing $\mathrm{pH}$, adsorption temperature and the initial $\mathrm{Pb}$ concentration. Under the condition of $\mathrm{pH}=7$ and temperature was $50^{\circ} \mathrm{C}$, the adsorption capacities reached the maximum. The adsorption equilibrium of raw-MWNTs on $\mathrm{Pb}$ reached balance in about 90min and the AO-MWNTs achieved stable in 60min. The adsorption process of AO-MWNTs could be better described by Freundlich model.
\end{abstract}

\section{Introduction}

Carbon nanotube(CNT) has been widely researched since it was found in 1991[1].Carbon nanotubes(CNTs) have seamless, hollow cylinder type structure and they were curly formed by one or multi-layer graphenes. With the unique mechanical and electrical performance, CNTs have broad application prospects in the field of electronics, optics and material[2].In respect of adsorption, CNTs with large specific surface area, the nano-scale pore structure and the surface can be easily modified, they can be widely used to act as adsorption materials[3].

But some bad characteristics of the CNTs greatly limit the application of themselves[4], such as the strong van der Waals force, high surface activity, very easy to reunite and can't be easily dispersed in aqueous solution. Chemical functional groups could be introduced to modified the surface properties of CNTs[5], so as to increase their hydrophilicity and strengthen the ability of removing harmful pollutants in aqueous solution. In this paper, amidoxime group was used to modified the multi-wall carbon nanotube(MWNTs), in order to enhance the dispersion of MWNTs, improve the adsorption properties for metal ions in aqueous solution, and provide a new effective technique for the treatment of heavy metal ions.

\section{Materials and Methods}

\section{Materials}

MWNTs: Shenzhen NANO Tech. Port. Co. Ltd. (Shenzhen, China), diameter 20-40nm, length $>5 \mathrm{~m}$, the purity was more than $97 \%$. Uranium standard solution: $1.1792 \mathrm{~g} \mathrm{U}_{3} \mathrm{O}_{8}$ was placed in a beaker of $100 \mathrm{~mL}$, added $10 \mathrm{~mL}$ hydrochloric acid, $3 \mathrm{~mL} \mathrm{H}_{2} \mathrm{O}_{2}$ and about two drops of nitric acid. After the severe reaction, heated the beaker until $\mathrm{U}_{3} \mathrm{O}_{8}$ completely dissolved, and then transferred the liquid to a $1000 \mathrm{~mL}$ volumetric flask, added distilled water to the scale and shook, per $1 \mathrm{~mL}$ this solution contained $1 \mathrm{~mL}$ uranium.

\section{Methods}

The $10.0 \mathrm{~g}$ raw multi-wall carbon nanotube(MWNTs) were refluxed in a strong oxidizing agent (3:1 concentrated $\mathrm{H}_{2} \mathrm{SO}_{4} / \mathrm{HNO}_{3}$ mixture)[6] at $60^{\circ} \mathrm{C}$ for $12 \mathrm{~h}$. Then $100 \mathrm{~mL}$ of distilled water was 
added and the mixture was vacuum-filtered through a $0.45 \mu \mathrm{m}$ pore size PTFE filter paper. The filtrate was then washed with distilled water until the eluate reached the neutral $\mathrm{pH}$ and dried at $50^{\circ} \mathrm{C}$ to obtain carboxylated CNTs(COOH-MWNTs).Second, 9.0g COOH-MWNTs(after granding) were added to a three-necked flask, $2 \mathrm{ml}$ dimethyl formamide (DMF) and 70ml dichloride sulfoxide $\left(\mathrm{SOCl}_{2}\right)$ were added, then heated to $70{ }^{\circ} \mathrm{C}$ and refluxed for $12 \mathrm{~h}$, with tetrahydrofuran (THF) centrifugal washing five times to remove the solvent to yielded acyl chloride-functionalized MWNTs (COCl-MWNTs).Thirdly, according to document[7], 8.0g COCl-MWNTs were added to $100 \mathrm{~mL} 25 \%$ ammonia under ice bath condition, then reacted $4 \mathrm{~h}$ in $10^{\circ} \mathrm{C}$ environment, through highspeed centrifugation to get rid of supernatant, washed several times with methanol, dried under $50^{\circ} \mathrm{C}$ and obtained the amide carbon nanotubes ( $\mathrm{NH} 2-\mathrm{MWNTs}$ ). Fourth, $6.0 \mathrm{~g} \mathrm{NH}_{2}-\mathrm{MWNTs}$ were added into the mixed solution of $200 \mathrm{ml}$ acetonitrile, $40 \mathrm{~mL} 37 \%$ formaldehyde and $40 \mathrm{~mL}$ acetic acid. Heated to $95^{\circ} \mathrm{C}$ and reacted $12 \mathrm{~h}$, then the mixture was vacuum-filtered through a $0.45 \mu \mathrm{m}$ pore size PTFE filter paper, the filtrate was washed with methanol. Vacuum drying under $50^{\circ} \mathrm{C}$ to obtain the cyano functionalized carbon nanotubes(CN-MWNTs).Afterward, $8.686 \mathrm{~g}$ hydroxylamine hydrochloride $\left(\mathrm{NH}_{2} \mathrm{OH} \cdot \mathrm{HCl}\right)$ and $250 \mathrm{~mL}$ distilled water were putted in a flat bottom flask, added $6.625 \mathrm{~g} \mathrm{Na}_{2} \mathrm{CO}_{3}$ while pumped in $\mathrm{N}_{2}$.Stirring and heating until they dissolved completely.Then 5.0 g CN-MWNTs was added and reacted under magnetic stirring and the temperature of $70^{\circ} \mathrm{C}$. Through the vacuum filtration, the filter residue was washed with distilled water several times and got the final product of amidoxime functionalized carbon nanotubes(AO-MWNTs) after dried under $50^{\circ} \mathrm{C}$.

\section{Adsorption Experiment}

Under the condition of different factors respectively investigated the adsorption effect of $\mathrm{Pb}$ on AO-MWNTs. After adsorbed completely, then vacuum-filtered through a $0.45 \mu \mathrm{m}$ pore size PTFE filter paper, the $\mathrm{Pb}$ concentration in filtrate was measured by the atomic absorption spectrophotometer.

\section{Results}

\section{Suspension Dispersion Stability}

Dispersion stability was an important index to evaluate the effect of the modification of carbon nanotubes, collision between the particles in dispersion liquid occurred due to the Brown movement, it was easy to generate attractive interaction between the particles, when attraction was less than repulsion, carbon nanotubes can be relatively stable dispersion in aqueous solution, on the contrary, precipitation was produced. A certain amount of different types of carbon nanotubes were separately dissolved in distilled water and were ultrasonic dispersed 30min, obtained mass concentration of $0.8 \mathrm{~g} / \mathrm{L}$ carbon nanotubes dispersion. The suspensions stabilities of different types of carbon nanotubes were listed in Table1, and the dispersed photos after 1day and 30days were separately shown in Fig.1.From this experiment could find that the liquid dispersion stabilities of COOH-MWNTs and AO-MWNTs were both well, while the stabilities of raw-MWNTs and $\mathrm{NH}_{2}-$ MWNTs were not good.

Tab. 1 The suspension dispersion stability of different CNTs

\begin{tabular}{cc}
\hline Carbon nanotube type & Precipitation time \\
\hline raw-MWNTs & Less than one hour \\
$\mathrm{NH}_{2}$-MWNTs & One day \\
COOH-MWNTs & No precipitation after 30 days \\
AO-MWNTs & No precipitation after 30 days \\
\hline
\end{tabular}




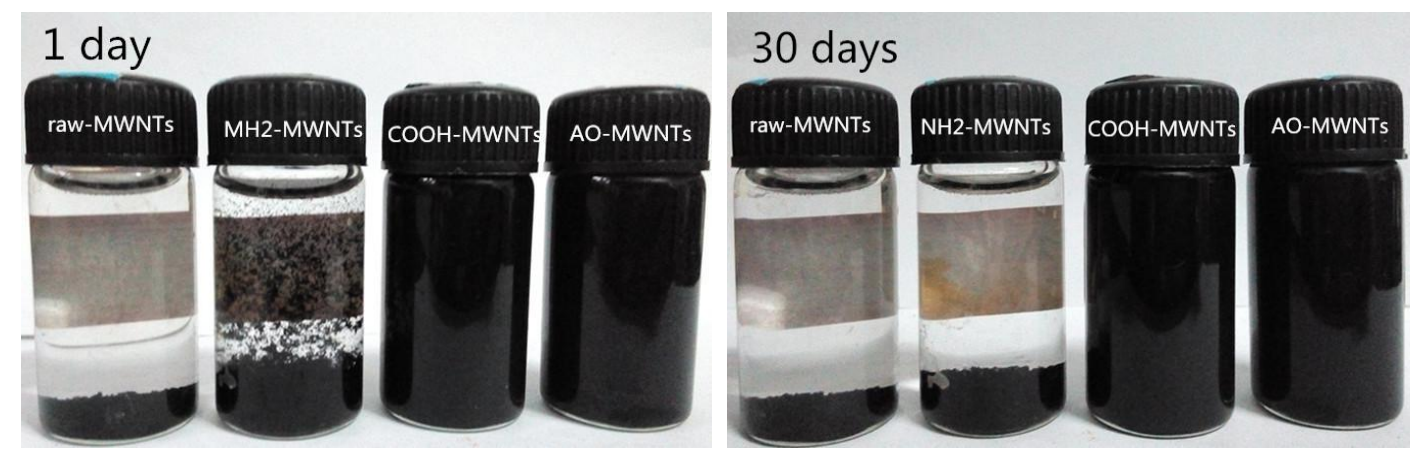

Fig.1 Different times of the suspension dispersed photos of CNTs

\section{FT-IR Spectroscopy}

Fig.2 showed the FT-IR spectra of raw-MWNTs(raw) and AO-MWNTs(AO).before modified, band of $1627 \mathrm{~cm}^{-1}$ was appeared, which was due to $\mathrm{C}=\mathrm{O}$ stretching vibration, and the strong wide band of $3440 \mathrm{~cm}^{-1}$ was belonged to the stretching vibration of $-\mathrm{OH}$.Observation can be found that AO-MWNTs appeared the band at $3282 \mathrm{~cm}^{-1}$, which corresponded to the stretching vibration of $-\mathrm{OH}$ in amidoxime group and the vibration of $-\mathrm{NH}_{2}$ in primary amine. The $\mathrm{C}=\mathrm{N}\left(1641 \mathrm{~cm}^{-1}\right)$ stretching vibration and the vibration of $\mathrm{N}-\mathrm{O}\left(931 \mathrm{~cm}^{-1}\right)$ could also be found, so amidoxime group was introduced to the carbon nanotubes.

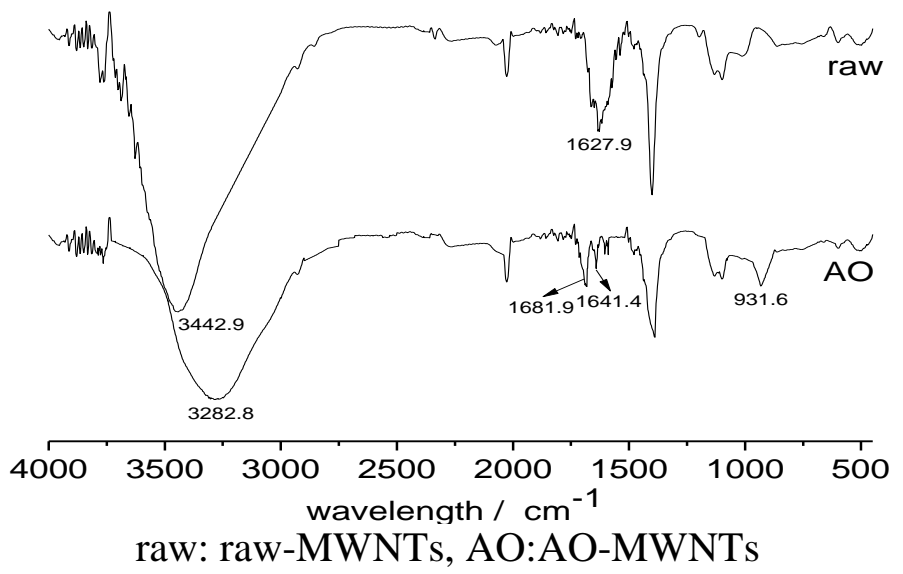

Fig.2 Infrared spectra of MWNTs

\section{Effect of pH}

The $10 \mathrm{mg} / \mathrm{L}$ simulated waste water of $\mathrm{Pb}$ was prepared, adsorbent dose was $0.5 \mathrm{~g} / \mathrm{L}$ and adjusted the $\mathrm{pH}$ in the range of $2-7$, under $25{ }^{\circ} \mathrm{C}$ conditions investigating the absorption influence of $\mathrm{pH}$ on amidoxime carbon nanotubes of $\mathrm{Pb}$. The adsorbents were ultrasonic dispersed $15 \mathrm{~min}$ by ultrasonic cleaning machine firstly, oscillated $1 \mathrm{~h}$ and vacuum-filtered through $0.45 \mu \mathrm{m}$ microporous membrane, the $\mathrm{Pb}$ concentration of filtrate was measured by atomic absorption spectrophotometer. When adjusting $\mathrm{pH}$ over $6, \mathrm{~Pb}$ gradually precipitated, this may influenced the determination results. While in the process of ultrasonic dispersion, the ultrasonic effect of raw-MWNTs was significantly less than AO-MWNTs.

The results were shown in Fig.3, with the increase of $\mathrm{pH}$, the adsorption capacities of $\mathrm{Pb}$ on rawMWNTs and AO-MWNTs were both increased gradually, it was because the solubility of $\mathrm{Pb}$ decreased while the $\mathrm{pH}$ value increased, white precipitate could be easily found when $\mathrm{pH}=7$, this may reduce the $\mathrm{Pb}$ concentration of filtrate, so in order to achieve the better adsorption of $\mathrm{Pb}$, the $\mathrm{pH}$ value was adjusted to 6 in the next experiment.

\section{Effect of Initial $\mathrm{Pb}$ Concentration}

Under the condition of $\mathrm{pH}=6$, adsorption dosage was $0.5 \mathrm{~g} / \mathrm{L}$, temperature was $25^{\circ} \mathrm{C}$, the initial concentration of $\mathrm{Pb}$ were $2,5,10,25,50,100 \mathrm{mg} / \mathrm{L}$ separately. The adsorbents were ultrasonic 
dispersed $15 \mathrm{~min}$ by ultrasonic cleaning machine firstly, oscillated $1 \mathrm{~h}$ and vacuum-filtered through $0.45 \mu \mathrm{m}$ microporous membrane, the $\mathrm{Pb}$ concentration of filtrate was measured by atomic absorption spectrophotometer. As shown in Fig.4, with the increase of initial $\mathrm{Pb}$ concentration, the adsorption capacities of raw-MWNTs and AO-MWNTs were both increased, but the Pb adsorption effect of AO-MWNTs was significantly better than raw-MWNTs, when initial $\mathrm{Pb}$ concentration was $100 \mathrm{mg} / \mathrm{L}$, the adsorption capacity of AO-MWNTs could reach $131.4 \mathrm{mg} / \mathrm{g}$, this better adsorption effect was due to the better dispersibility of AO-MWNTs.

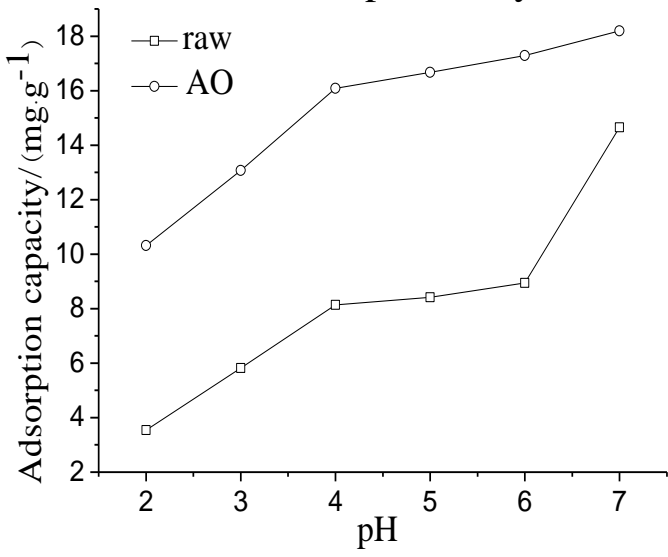

Fig.3 Effect of $\mathrm{pH}$ on adsorptivity

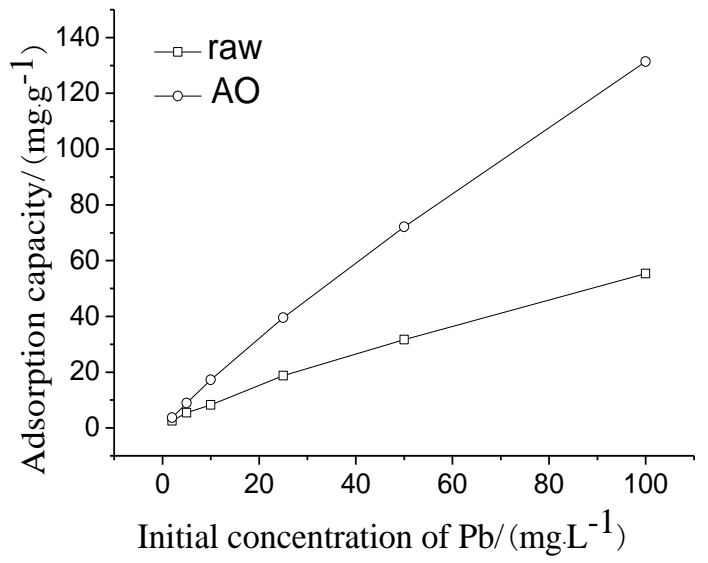

Fig 4 Effect of initial $\mathrm{Pb}$ concentration on adsorptivity

\section{Effect of Oscillating Time}

When the $\mathrm{pH}$ value was 6 , adsorbent dosage was $0.5 \mathrm{~g} / \mathrm{L}$, the concentration of $\mathrm{Pb}$ was $10 \mathrm{mg} / \mathrm{L}$, under the condition of the temperature of $25^{\circ} \mathrm{C}$ to examine the influence of oscillating time on the adsorption capacity. Firstly the adsorbents were ultrasonic dispersed $15 \mathrm{~min}$, oscillated and vacuumfiltered through $0.45 \mu \mathrm{m}$ microporous membrane, the residual $\mathrm{Pb}$ concentration of filtrate was measured by atomic absorption spectrophotometer. The results were shown in Fig.5, the adsorption capacity of AO-MWNTs on Pb achieved a balance after 30min, while the adsorbing material of raw-MWNTs reached a steady after 60min.

\section{Effect of Temperature}

At $\mathrm{pH}=6, \mathrm{~Pb}$ concentration was $10 \mathrm{mg} / \mathrm{L}$, adsorbent dosage was $0.5 \mathrm{~g} / \mathrm{L}, 15 \mathrm{~min}$ ultrasonic dispersed and oscillated $1 \mathrm{~h}$ to investigated the influence that the temperature made on the adsorption process. From Fig. 6 can obtained, the adsorption capacities of AO-MWNTs and rawMWNTs were both gradually increased with the increasing of temperature, the $\mathrm{Pb}$ adsorption process of this two adsorbents belonged to endothermic reaction, increasing temperature could make contribution to the process of $\mathrm{Pb}$ adsorption.

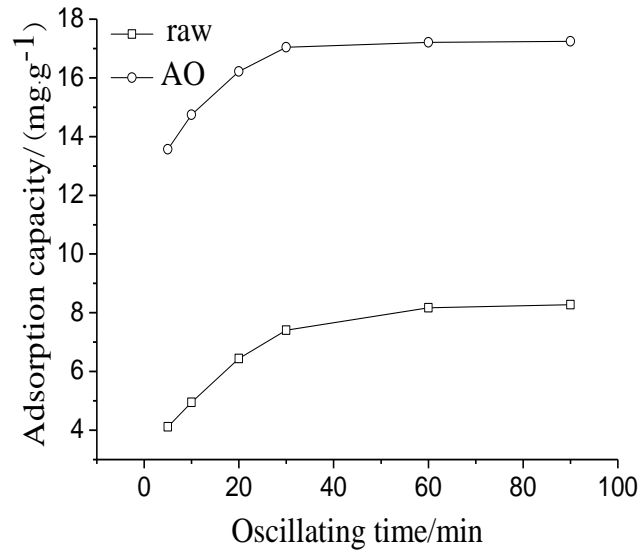

Fig.5 Effect of oscillating time on adsorptivity

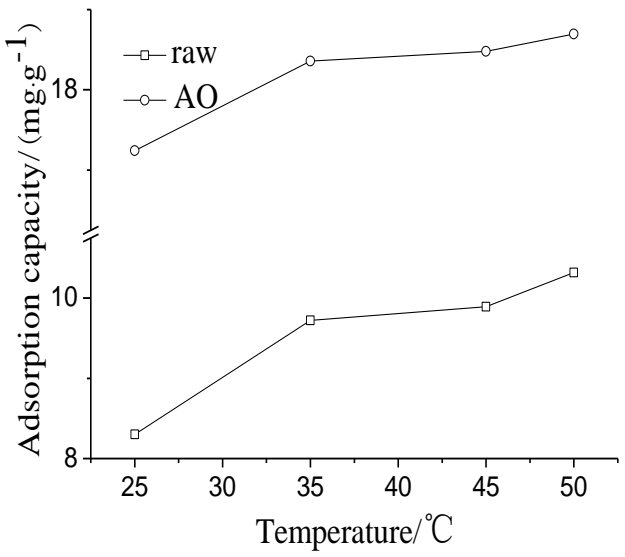

Fig.6 Effect of temperature on adsorptivity 


\section{Isothermal Adsorption Model}

The adsorption isotherm was described the changing characteristics between adsorption capacity of the adsorbent and adsorbate concentration when reached the adsorption equilibrium under certain temperatures. In this paper, Langmuir and Freundlich model were used to fit the adsorption process of AO-MWNTs on Pb.The results were respectively shown in Fig.7 and Fig.8, the concluded relevant datas were listed in Table 2.From the simulations of this two models to the adsorption process could be seen, the concluded relevant data $\mathrm{R}^{2}$ of the Freundlich adsorption isotherm model to simulate the adsorption process of AO-MWNTs was more than $99 \%$, better than the Langmuir fitting degree, so the adsorption type of AO-MWNTs on Pb could be better described by Freundlich model, the multilayer adsorption was the main type.

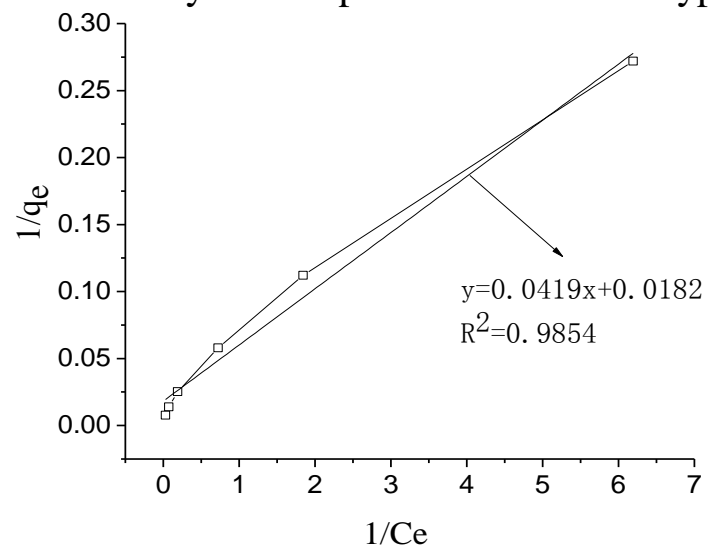

Fig.7 Fitting line of Langmuir adsorption isotherm

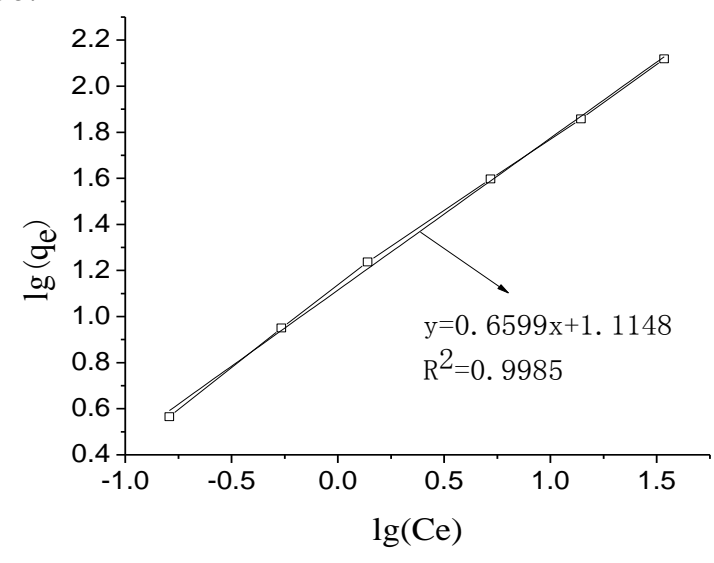

Fig.8 Fitting line of Freundlich adsorption isotherm

Tab. 2 Values of Langmuir and Freundlich constants of AO-MWNTs on the adsorption of $\mathrm{Pb}$

\begin{tabular}{ccccccc}
\hline \multicolumn{3}{c}{ Langmuir } & \multicolumn{3}{c}{ Freundlich } \\
\hline & $\mathrm{q}_{\max }$ & $\mathrm{b}$ & $\mathrm{R}^{2}$ & $1 / \mathrm{n}$ & $\mathrm{k}$ & $\mathrm{R}^{2}$ \\
$\mathrm{AO}$ & 54.945 & 0.4344 & 0.9854 & 0.6653 & 3.0158 & 0.9985 \\
\hline
\end{tabular}

\section{Conclusions}

In this work, the cyano group was firstly introduced on CNTs, then amidoxime group was used to modifiy the CN-MWNTs. The detection method of FTIR proved that a novel adsorption material(AO-WMNTs) was produced.This methods improved the dispersion of carbon nanotubes in aqueous solution and enhanced the adsorption capacity of $\mathrm{Pb}$. The adsorption experiments showed that: the $\mathrm{Pb}$ adsorption capacities of raw-MWNTs and AO-MWNTs were both gradually increased with the increasing of the $\mathrm{pH}$ value, adsorption temperature and initial concentration of $\mathrm{Pb}$. Results also obtained that AO-MWNTs could be used as a good $\mathrm{Pb}$ adsorption material, the adsorption capacity of $\mathrm{Pb}$ on it was better than raw-MWNTs, and the time it needed to reach the balance was shorter than raw-MWNTs. So, this experiment can make contribution to the better use of carbon nanotubes and provide a better treatment of heavy metal ions.

\section{Acknowledgements}

This work was financially supported by the National Natural Science Foundation of China(No.11275095) and Science Program of Hunan Environmental Protection Department(Xiangcai.2012-347).

\section{References}

[1] S. Iijima. Helical microtubules of graphitic carbon [J]. Nature, 1991, 354: $56 \sim 58$. 
[2] LIU Boliang, ZHANG Yujun, WANG Pufang, et al.Removal of copper ( II) from aqueous solution by carbon nanotubes[J].Applied Chemical Industry, 2011, 40(1):68-70.

[3] Machado, F. M., Bergmann, C. P., Fernandes, T. H. M., et al. Adsorption of Reactive Red M2BE dye from water solutions by multi-walled carbon Nanotubes and activated carbon[J]. Journal of Hazardous Materials, 2011, 192 (3):1122-1131.

[4] Xu LiHeng, Zhang Ming, Chen Feng.Construction of CNTs-based composite adsorbents and their adsorption Capacity[J].Acta Scientiae Circumstantiae, 2014, 34(6):1443-1448.

[5] LI Bo, LIANG Yongfu, SHI Zujin, et al.Chemical Modification of single carbon nantotubes[J]. Journal of high school chemistry, 2000, 21:1633-163.

[6] ZHANG Binbin, SHEN Jianfeng, HU Yizhe, et al.Functionalization of multi-walled carbon nanotubes using diamines[J].Journal of FudanUniversity, 2008, 47(4):454-460.

[7] Gao Wenhua, Sun Ximeng, Chen Tufeng, et al.Preparation of cyano-Functionalized multiwalled carbon nanotubes as solid-phase extraction sorbent for preconcentration of phenolic compounds in environmental water[J]. J.Sep.Sci., 2012, 35:1967-1976. 